\title{
Experimental test of reciprocity relations in quantum thermoelectric transport
}

\author{
J. Matthews, ${ }^{1}$ F. Battista, ${ }^{2,3}$ D. Sánchez ${ }^{4}$ P. Samuelsson, ${ }^{2,3}$ and H. Linke ${ }^{2,3}$ \\ ${ }^{1}$ Physics Department and Materials Science Institute, \\ University of Oregon, Eugene, Oregon 97403-1274 \\ ${ }^{2}$ Physics Department, Lund University, Box 118, S-221 00, Lund, Sweden \\ ${ }^{3}$ Nanometer Structure Consortium (nmC@LU), Lund University, Box 118, S-221 00, Lund, Sweden \\ ${ }^{4}$ Instituto de Física Interdisciplinar y Sistemas Complejos IFISC (CSIC-UIB), E-07122 Palma de Mallorca, Spain
}

\begin{abstract}
Fundamental symmetries in thermoelectric quantum transport, beyond Onsagers relations, were predicted two decades ago but have to date not been observed in experiments. Recent works have predicted the symmetries to be sensitive to energy-dependent, inelastic scattering, raising the question whether they exist in practice. Here we answer this question affirmatively by experimentally verifying the thermoelectric reciprocity relations in a four-terminal mesoscopic device where each terminal can be electrically and thermally biased, individually. The linear response thermoelectric coefficients are found to be symmetric under simultaneous reversal of magnetic field and exchange of injection and emission contacts. We also demonstrate a controllable breakdown of the reciprocity relations by increasing thermal bias, putting in prospect enhanced thermoelectric performance.
\end{abstract}

PACS numbers:

\section{INTRODUCTION}

Symmetry relations are manifestations of fundamental principles and constitute cornerstones of modern physics. A prominent example is the Onsager relations $\frac{1}{1}$ between coefficients connecting thermodynamic fluxes and forces, which derive from the principle of microreversibility. In the quantum transport regime, Onsagers relations for electrical resistance ${ }^{2}$ have been observed in multiterminal mesoscopic systems 3 .4 In addition to the Onsager relations, reciprocity relations for thermoelectric (TE) transport coefficients were predicted ${ }^{5,6}$ : reversing the magnetic field and simultaneously exchanging the injection and emission contacts is expected to leave the coefficients invariant.

In addition to their fundamental interest, the reciprocity relations are of practical importance. On the one hand, the existence of symmetry relations could simplify the theory of improved, future TE materials, such as nanoscale, anisotropic $\underline{7.8}$ or hybrid materials $\underline{\underline{9}}$ where nonlocal effects may play a role. On the other hand, the absence of symmetries could be equally important: asymmetric thermopower was recently shown to allow for improved TE performance ${ }^{10}-12$ in the maximum power regime.

However, to date the reciprocity relations have not been tested experimentally, and the extent to which they can be observed is unclear. Recent works ${ }^{13}-15$ theoretically investigated the robustness of magnetic field symmetries in the thermopower, which are directly related to the thermoelectric reciprocity relations. In contrast to Onsagers relations ${ }^{16}$, it was predicted that inelastic electron scattering (always present at finite temperature), in combination with a breakdown of the WiedemannFranz law can break the thermopower symmetries. The Wiedemann-Franz law is known to break down in lowdimensional structures due to their strongly energydependent density of states $^{17}$ - the same property that makes them interesting candidates for TE-materials 18 .

A fundamental question is thus: can TE reciprocity relations be observed in practice and can they be controlled in experiment? Such a test of the TE reciprocity relations requires a multi-terminal normal conductor where each terminal can be electrically and thermally biased, individually, while subjected to an applied magnetic field.

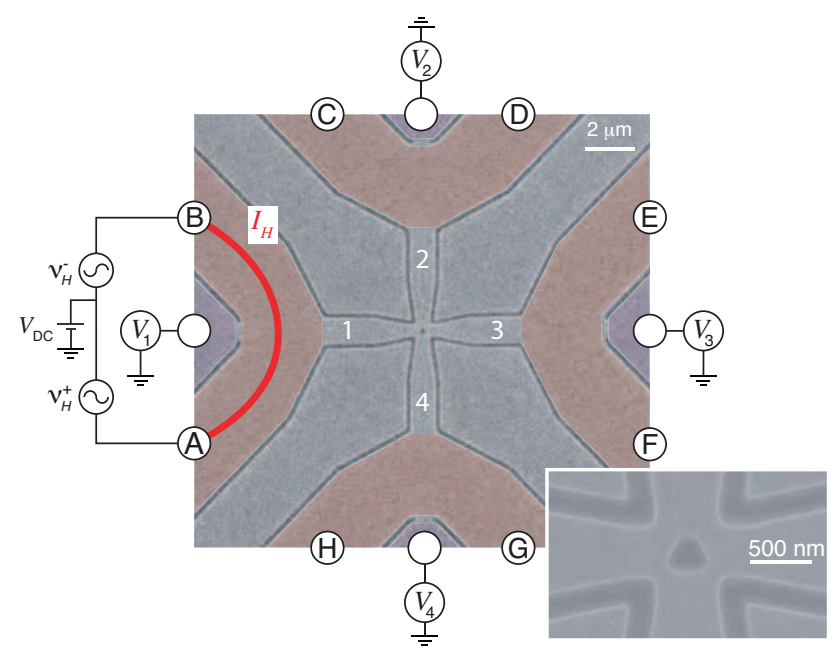

FIG. 1: (Color online) Scanning electron micrograph of a device identical to the one measured on here, featuring a junction of four ballistic micro-channels (terminals) in a cross configuration, with an asymmetric scatterer in the central junction. The eight surrounding contacts, $\{\mathrm{A}, \mathrm{B}, \ldots \mathrm{H}\}$, are used to apply a thermal or electrical bias. Four probes are used to measure the terminal voltages: $\left\{V_{1}, V_{2}, V_{3}, V_{4}\right\}$. The regions between contact pairs, tinted red, can be electrically heated to thermally bias the junction. In the configuration shown, the channel between contacts $\mathrm{A}$ and $\mathrm{B}$ are heated through two out-of-phase heating voltages, $\nu_{H}^{ \pm}$(see Appendix A). (Inset) Close up image of the central region.

Here we present such an experimental test in a four- 
terminal mesoscopic device (Fig. 1), and establish that the TE reciprocity relations manifest themselves in real devices. We also find evidence for a breakdown of the relations when we increase the thermal bias, indicating that the symmetries can be experimentally controlled, either by inelastic scattering or by non-linear thermal transport, analogous to the symmetry-breakdown in purely electronic transport at finite voltages in mesoscopic systems $\underline{19}-26$. This motivates further investigations on the symmetry breaking properties and relative role of inelastic scattering and non-linear thermal transport.

\section{SYSTEM AND METHOD}

We first spell out the properties of the four terminal device. It was defined by patterning the two-dimensional electron gas (2DEG) formed in an $\mathrm{InP} / \mathrm{Ga}_{0.23} \mathrm{In}_{0.77} \mathrm{As}$ heterostructure by using electron-beam lithography and shallow wet etching (for details, see $\operatorname{Ref} \underset{\underline{\underline{T}}}{ }$ ). The wafer has a carrier concentration of $n=1.1 \times 10^{12} \mathrm{~cm}^{-2}$ and an electron mobility of $\mu=3.2 \times 10^{5} \mathrm{~cm}^{2} / \mathrm{Vs}$ at $4.2 \mathrm{~K}$. Moreover, all measurements were made in a $\mathrm{He} 3$ cryostat at a background temperature of $\theta_{0}=240 \mathrm{mK}$. To create a thermal bias, we used heating voltages of typically $V_{H}=400 \mu \mathrm{V}$ (unless stated otherwise), resulting in heating currents of less than $400 \mathrm{nA}$ and an estimated electric heating power of less than $0.1 \mathrm{nW}$ delivered to the heating area, resulting in an estimated temperature rise $\theta<1$ $K$. The stray heating power due to thermal conductance to neighboring heating pads was negligible. Moreover, we checked carefully that the electric bias measurements were in the linear response regime (see Appendix A for details on device properties).

We proceed by defining the thermoelectric coefficients and their expected symmetry, and describe how they can be determined in experiments. The linear response of the electrical current flowing in the $\alpha^{\prime}$ th terminal, $I_{\alpha}$, of a multi-terminal, mesoscopic junction is

$$
I_{\alpha}=\sum_{\beta \neq \alpha}\left[G_{\alpha \beta}\left(V_{\alpha}-V_{\beta}\right)+L_{\alpha \beta}\left(\theta_{\alpha}-\theta_{\beta}\right)\right],
$$

where $V_{\alpha}$ and $\theta_{\alpha}$ are the voltage and temperature, respectively, at terminal $\alpha$, and $G_{\alpha \beta}$ and $L_{\alpha \beta}$ are the electrical conductance and thermoelectric coefficients, respectively, between terminals $\alpha$ and $\beta$. The $L_{\alpha \beta}$ are directly related to the thermopower, or Seebeck coefficients $\mathcal{S}_{\alpha \beta} \equiv$ $\left(V_{\alpha}-V_{\beta}\right) /\left.\left(\theta_{\alpha}-\theta_{\beta}\right)\right|_{I=0}$, through $L_{\alpha \beta}=-\sum_{\gamma} G_{\alpha \gamma} \mathcal{S}_{\gamma \beta}$, where the sum runs over all terminals ${ }^{5}$.

The transport properties in open mesoscopic systems can conveniently be described by the scattering approach. The conductance coefficients, see Eq. (11), are given by $\underline{\underline{2}}$

$$
G_{\alpha \beta}(B)=\frac{2 e^{2}}{h} \int d E\left(-\frac{d f(E)}{d E}\right) T_{\alpha \beta}(E, B),
$$

for $\alpha \neq \beta$, where $T_{\alpha \beta}(E, B)$ is the transmission function describing scattering of particles at energy $E$ from terminal $\beta$ to $\alpha, B$ is the magnetic field, and $f(E)$ is the equilibrium Fermi distribution function. Correspondingly, the thermoelectric coefficients are given by ${ }^{\underline{5}}$

$$
L_{\alpha \beta}(B)=\frac{2 e}{h \theta_{0}} \int d E E\left(-\frac{d f(E)}{d E}\right) T_{\alpha \beta}(E, B),
$$

where we have set the background chemical potential to zero.

Microreversibility demands that the transmission function obeys the magnetic-field symmetry $T_{\alpha \beta}(E, B)=$ $T_{\beta \alpha}(E,-B)$. The resulting symmetry for the conductance, $G_{\alpha \beta}(B)=G_{\beta \alpha}(-B)$, has been thoroughly investigated over the last few decades $2-\underline{\underline{4}}$. Since the thermoelectric coefficients depend directly on the transmission function, $L_{\alpha \beta}$ should obey the same symmetry properties as $G_{\alpha \beta}$. This gives, writing out the diagonal and off-diagonal relations separately,

$$
L_{\alpha \alpha}(B)=L_{\alpha \alpha}(-B), \quad L_{\alpha \beta}(B)=L_{\beta \alpha}(-B) .
$$

To experimentally test these symmetries, we first determine $G_{\alpha \beta}$ through electric bias measurements with no thermal bias $\left(\Delta \theta_{\alpha \beta}=0\right)$ (see Appendix B). Thereafter, the thermoelectric coefficients are investigated by thermally biasing the system under zero-electric-current conditions (with floating terminals), measuring the resulting potentials in all reservoirs, and using Eq. (1D) as explained in the following.

The induced temperature increase at terminal $\alpha$ can be written as a Fourier sum, $\Delta \theta_{\alpha}(t) \equiv \theta_{\alpha}(t)-\theta_{0}=$ $\sum_{n=0} \Delta \theta_{\alpha}^{(n)} \sin (n \omega t)$, where $\omega$ is the frequency of the heating current. This allows us to write the different Fourier components of the linear response current expression in Eq. (11) as

$$
0=\sum_{\beta}\left[G_{\alpha \beta} V_{\alpha \beta}^{(n)}+L_{\alpha \beta} \theta_{\alpha \beta}^{(n)}\right],
$$

where $V_{\alpha \beta}^{(n)} \equiv V_{\alpha}^{(n)}-V_{\beta}^{(n)}$, and $\theta_{\alpha \beta}^{(n)} \equiv \theta_{\alpha}^{(n)}-\theta_{\beta}^{(n)}=$ $\Delta \theta_{\alpha}^{(n)}-\Delta \theta_{\beta}^{(n)}$. When using Joule heating (quadratic in heating current), one expects the second harmonic to give the strongest contribution to the thermoelectric response. Indeed, in our experiment we find that $n=2$ gives the largest signal and provides the clearest data to determine the range of the linear response regime; in the following, we only consider the second harmonics in Eq. (5). Heating the $\gamma^{\prime}$ th terminal, and making the assumption that the unheated terminals remain cold, we can make use of the relation $\sum_{\alpha} L_{\alpha \beta}=\sum_{\beta} L_{\alpha \beta}=0$, which follows from the unitarity of the scattering matrix ${ }^{5}$, and write

$$
L_{\alpha \gamma} \Delta \theta_{\gamma}^{(2)}=\sum_{\beta} G_{\alpha \beta} V_{\alpha \beta}^{(2)} .
$$

Here, $V_{\alpha \beta}^{(2)}$ represents the measured values when heating terminal $\gamma$. Since the $G_{\alpha \beta}$ elements depend weakly on magnetic field up to $B \sim 50 \mathrm{mT}$ (see Appendix B), we 
can use Eq. (6) and $G_{\alpha \beta}$ to test the magnetic-field symmetries of $L_{\alpha \gamma} \Delta \theta_{\gamma}^{(2)}$ directly by analysing the $B$-field dependence of the measured $V_{\alpha \beta}^{(2)}$. In the following, we also assume that $\Delta \theta_{\gamma}^{(2)}$ is independent of $B$, so that all of the $B$-field dependence in $L_{\alpha \gamma} \Delta \theta_{\gamma}^{(2)}$ comes from $L_{\alpha \gamma}$.

\section{THERMOELECTRIC RECIPROCITY RELATIONS}

The symmetry relations predicted by Eq. (4) are clearly visible in the representative magnetic field traces for $L_{\alpha \gamma} \Delta \theta_{\gamma}^{(2)}$ presented in Fig. 2b),c). We also find that there is no significant symmetry relation between $L_{\alpha \beta}(B)$ and $L_{\gamma \delta}(-B)$ for $\alpha \beta \neq \gamma \delta$ (see Fig. 2 $\mathrm{d}$ ) for an example). The $L_{\alpha \gamma} \Delta \theta_{\gamma}^{(2)}$ typically oscillate around zero, a signature of quantum interference effects $27-29$.

To quantify the degree of symmetry, we make use of the correlation coefficient ${ }^{30}$ between $L_{\alpha \beta}(B)$ and $L_{\gamma \delta}(-B)$ (see Appendix C). We first introduce the normalized thermoelectric coefficients

$$
\mathcal{L}_{\alpha \beta}(B) \equiv \frac{L_{\alpha \beta}(B)-\left\langle L_{\alpha \beta}(B)\right\rangle}{\sqrt{\left\langle\left[L_{\alpha \beta}(B)\right]^{2}\right\rangle-\left\langle L_{\alpha \beta}(B)\right\rangle^{2}}},
$$

where $\langle\ldots\rangle$ denotes the average over magnetic fields from $-50 \mathrm{mT}$ to $50 \mathrm{mT}$. This magnetic field range was chosen to avoid the onset of classical commensurability effects. We calculate Eq. (7) using $L_{\alpha \beta} \Delta \theta^{(2)}$ in place of $L_{\alpha \beta}$, since we have assumed that $\Delta \theta^{(2)}$ is independent of $B$ and thus cancels out. We then define the symmetry parameter as

$$
\Sigma_{\alpha \beta, \gamma \delta} \equiv\left\langle\mathcal{L}_{\alpha \beta}(B) \mathcal{L}_{\gamma \delta}(-B)\right\rangle
$$

Note that $\Sigma_{\alpha \beta, \gamma \delta}$ goes from +1 for complete symmetry, to -1 for complete anti-symmetry. We stress that $\Sigma_{\alpha \beta, \gamma \delta}$ is well suited to quantify the overall symmetry of functions which, like the ones in Fig. 2, display rapid oscillations on top of a smooth, slowly oscillating background. The need for a quantitative symmetry measure becomes apparent when comparing Figs. 2b) and 2r); while the corresponding symmetry parameters are essentially identical, $\Sigma_{44,44} \approx \Sigma_{24,42}$, to the bare eye the curves in Fig. $2 \mathrm{~b})$ appear more symmetric than the ones in 2r). We have analyzed all combination of curves $\alpha \beta, \gamma \delta$ and also compared the results to another potential symmetry measure, the magnitude of the fluctuation of the difference, $\left\langle\left[\mathcal{L}_{\alpha \beta}(B)-\mathcal{L}_{\gamma \delta}(-B)\right]^{2}\right\rangle$. The result (not presented here) firmly establishes that $\Sigma_{\alpha \beta, \gamma \delta}$ is a reliable symmetry measure.

Deviations from the perfect symmetries predicted in Eq. (41) are seen in our measurements. To rule out noise as the cause of the limited symmetries, we verified that two traces measured almost two weeks apart showed very high correlation (see Appendix C), demonstrating the high repeatability of these fluctuations. We attribute
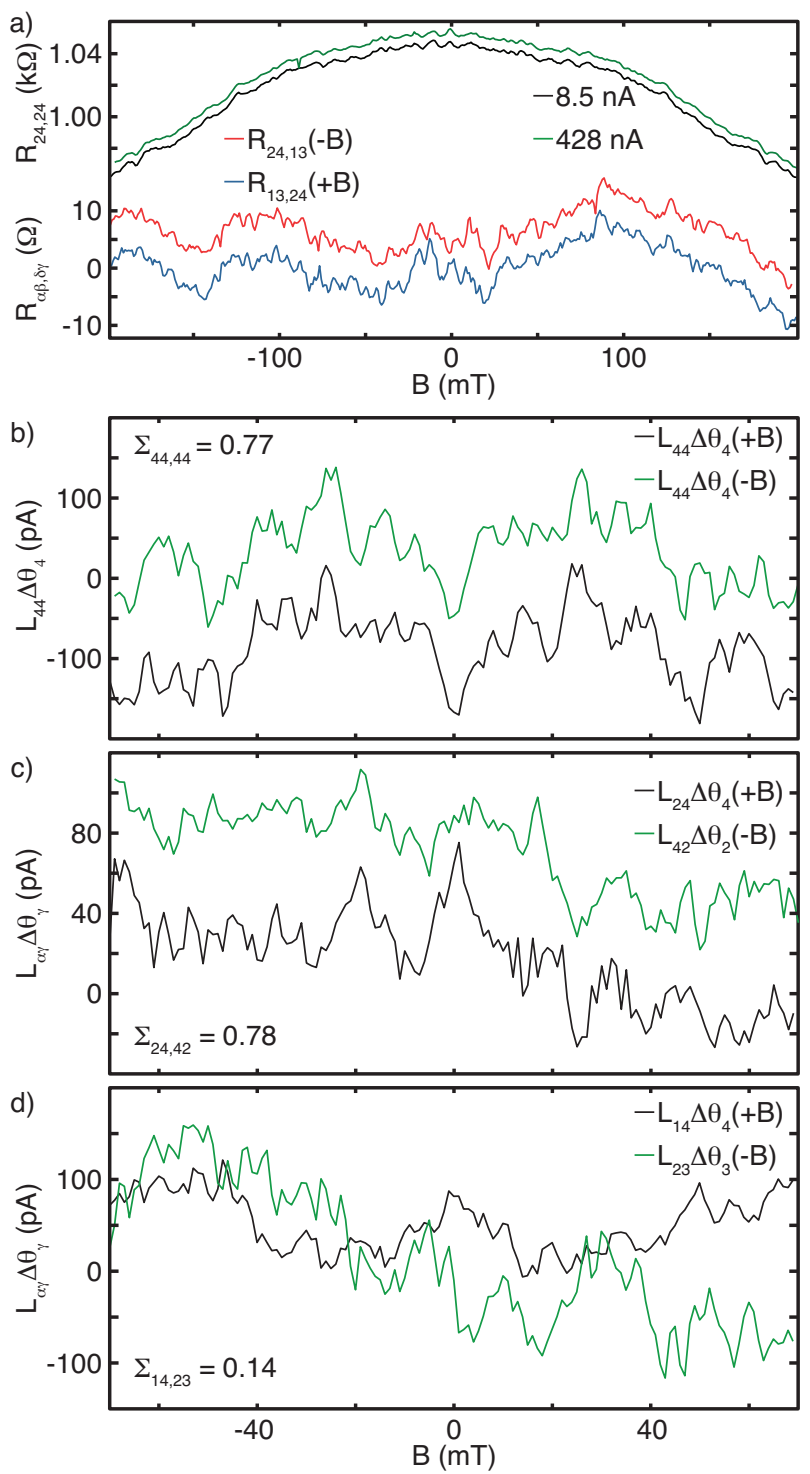

FIG. 2: (Color online) a) Representative four-terminal resistances $R_{\alpha \beta, \gamma \delta}(B)$ as a function of magnetic field. The symmetry $R_{\alpha \beta, \gamma \delta}(B)=R_{\gamma \delta, \alpha \beta}(-B)$ is clearly visible; small deviations are discussed in Appendix B. Note that the resistance $R_{24,24}(B)$ depends weakly on magnetic field for $-50 \mathrm{mT}<$ $B<50 \mathrm{mT}$. b)-d) Magnetic field traces of the thermoelectric coefficient $L_{\alpha \beta}(B)$. Each panel also displays the corresponding quantitative symmetry parameter $\Sigma_{\alpha \beta, \gamma \delta}$ [Eq. (8)] calculated for the range $-50 \mathrm{mT}<B<50 \mathrm{mT}$, for the respective pair of traces shown. The symmetry of the diagonal terms $L_{\alpha \alpha}(B)=L_{\alpha \alpha}(-B)$ is clearly visible in b), as well as that of the off-diagonal terms $L_{\alpha \beta}(B)=L_{\beta \alpha}(-B)$, in c). For comparison, an example of the expected absence of symmetries, here between $L_{14}(B)$ and $L_{23}(-B)$, is illustrated in d) and manifested by a $\Sigma_{14,13}$ value near zero. The green and red curves in a), b), and c) are offset for clarity. All measurements were performed at a cryostat temperature of $\theta_{0}=240$ $\mathrm{mK}$. 


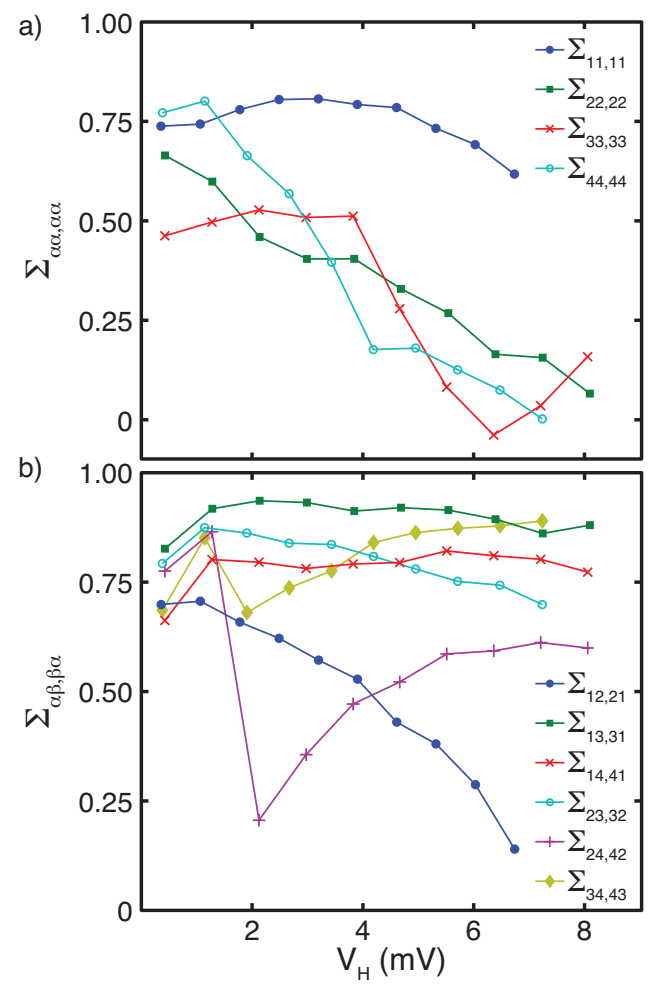

FIG. 3: (color online) Heating voltage dependence of the symmetries described in Eq. (4) for the a) diagonal, $\alpha=\beta$, and b) off-diagonal, $\alpha \neq \beta$, elements of $L_{\alpha \beta}$. In panel a), a clear trend for decreasing symmetry with increasing thermal bias is seen for the diagonal elements. This same trend is only present in three of the six curves in panel b).

the limited symmetry in Fig. 2b),c) mainly to the same mechanisms that limit the observed conductance symmetry. In addition, however, we offer two other possible mechanisms: i) the unheated terminals do not remain cold, which would modify Eq. (6); and ii) inelastic scattering, which at finite temperature can lead to asymmetries in the thermopower even in the linear response regime $\underline{13} \underline{\underline{15}}$.

\section{SYMMETRY BREAKDOWN}

One can expect the symmetry of $L_{\alpha \beta}$ to break down for finite heating voltage, analogous to the wellestablished breakdown of symmetries in the differential conductance $\frac{20,21}{2}$ observed at finite bias voltage in mesoscopic systems $19,22-26$. In Fig. 3, all ten symmetry relations defined by Eq. (4) are plotted as a function of heating voltage $V_{H}$. At low $V_{H}$, all symmetries described by Eq. (4) manifest themselves, with $\Sigma_{\alpha \beta, \beta \alpha} \gtrsim 0.5$. As $V_{H}$ is increased though, the trend in the diagonal elements, $\alpha=\beta$, is towards decreased symmetry, while the offdiagonal elements, $\alpha \neq \beta$, remain fairly symmetric with a slight trend to decrease.

The overall tendency is for the $B$-field symmetries of
$L_{\alpha \beta}$ to be suppressed with increasing thermal bias. From further analysis of our measured data (see Appendix D), we establish that the linear-response regime extends to about $V_{H} \approx 1 \mathrm{mV}$. The decreasing symmetry observed in $L_{\alpha \alpha}$, Fig. (3a), is then consistent with symmetry-breaking due to non-linear thermoelectric behavior ${ }^{31}-33$, analogous to non-linear electronic effects. Increased inelastic scattering due to heating effects may also play a role.

Further theoretical as well as experimental investigations are needed to explain the observed difference in symmetry between diagonal and off-diagonal elements of $L_{\alpha \beta}$ for $V_{H} \geq 1 \mathrm{mV}$, see Fig. 3. In particular, in contrast to linear response theory ${ }^{\underline{5}}$, existing non-linear theory $\underline{\underline{31}} \underline{\underline{33}}$ does not predict any simple relations between diagonal and off-diagonal elements.

\section{CONCLUSION}

In conclusion, we have verified that the TE reciprocity relations predicted more than 20 years $\operatorname{ago}^{\underline{\underline{5}}}$ manifest themselves in a mesoscopic device in the linear transport regime. The relations were observed at low temperatures, where inelastic scattering (predicted to suppress the symmetries ${ }^{13}-15$ ) can be expected to be small. At finite thermal bias we observe a breakdown of the reciprocity relations, tentatively due to a combination of inelastic scattering and non-linear thermal transport. Further investigations are needed to quantify the robustness of the reciprocity relations with respect to these mechanisms. Of particular interest will be the role of sharp features in the transmission function or density of states commonly used for energy filtering to enhance thermoelectric performance, for example in low dimensional coolers $\$ 4.35$ and highly efficient thermoelectric generators 36,37 . The possibility to experimentally control the absence or presence of the TE symmetry relations opens for exciting and fundamentally new opportunities in increasing TE energy efficiency $\underline{\underline{10}} \underline{-12}$.

\section{ACKNOWLEDGEMENTS}

We acknowledge financial support from NSF IGERT grant No. DGE-0549503, the National Science Foundation Grant No. DGE-0742540, ARO Grant No. W911NF0720083, nmC@LU, the ESF Research Network EPSD, the Foundation for Strategic Research (SSF), the Swedish Energy Agency (project number 38331-1), and MINECO Grant No. FIS2011-23526. Effort sponsored by the Air Force Office of Scientific Research, Air Force Material Command, USAF, under grant number FA865511-1-3037. The U.S. Government is authorized to reproduce and distribute reprints for Governmental purposes notwithstanding any copyright notation thereon. 


\section{Appendix A: Measurement of transport coefficients}

We individually use electric or thermal biases to determine the elements of the electrical conductance matrix, $G_{\alpha \beta}$, and the thermoelectric matrix, $L_{\alpha \beta}$, respectively. Electric biases are generated by applying a $37 \mathrm{~Hz}$ drive current between any two of the four central terminals. The measured $G_{\alpha \beta}$ are discussed below. A representative circuit configuration used for thermal-bias measurements is shown to the left in Fig. 1 in the main text. The thermal bias is generated by individually heating one terminal by applying two $180^{\circ}$ out-of-phase, $37 \mathrm{~Hz}$ voltages, denoted by $\nu_{H}^{ \pm}$, to the two channel contacts (in Fig. 1, main text, contacts $\mathrm{A}$ and $\mathrm{B}$ are used to heat terminal 1). This heating configuration is designed to eliminate any electric bias of the terminal due to the heating current. Additionally, a DC shift was applied to the thermal bias to cancel out residual DC offsets measured at the respective terminal's voltage probe. We note that only one terminal is heated at a time, and that negligible electric current is drawn through the junction during thermovoltage measurements. For further details on the thermal bias measurements, see Ref. [7]. Under each type of bias, all four-terminal voltages, $\left\{V_{1}, V_{2}, V_{3}, V_{4}\right\}$, were simultaneously measured using lock-in detection.

For thermal biasing, one pad was electrically heated using AC heating currents of between 300-400 nA at a frequency of $37 \mathrm{~Hz}$. The externally measured, two-terminal resistance of heating pads was between $1000-1400 \Omega$, such that $1 \mathrm{k} \Omega$ is a reasonable upper limit of the resistance of the heating pad itself. This gives an upper limit of the electric heating power delivered to a heating pad of about $0.1 \mathrm{nW}$. Finite-element simulations (COMSOL) that use the actual device geometry and take into account heat leaks to the surrounding 2DEG (but neglect additional heat leaks to the phonons) predict an upper limit of a temperature rise $\theta \approx 0.5-1 \mathrm{~K}$. Using the upper limit $\theta=1 \mathrm{~K}$, and estimating the thermal conductance between two heating pads (based on the electrical resistance between two pads of typically $1 \mathrm{k} \Omega$ and using the Wiedemann-Franz law), and again neglecting other heat leaks such as electron-phonon coupling, an upper limit for the resulting heating power to neighboring heat pads is about $6 \times 10^{-3} \mathrm{nW}$, about 100 times smaller than the intentional heating.

\section{Appendix B: Conductance measurements}

To determine the conductance coefficients at $B=0$, three different biasing configurations were utilized; each had the $37 \mathrm{~Hz}$ bias current injected at terminal 1, which was then extracted at terminals 2, 3 and 4 for the three biasing configurations. The rms amplitude of the bias current, $I$, was varied from 4 to $980 \mathrm{nA}$ to check for linearity in the current-voltage characteristics, sec Fig. 4 for a set of representative curves.

We stress that for the entire bias current range used

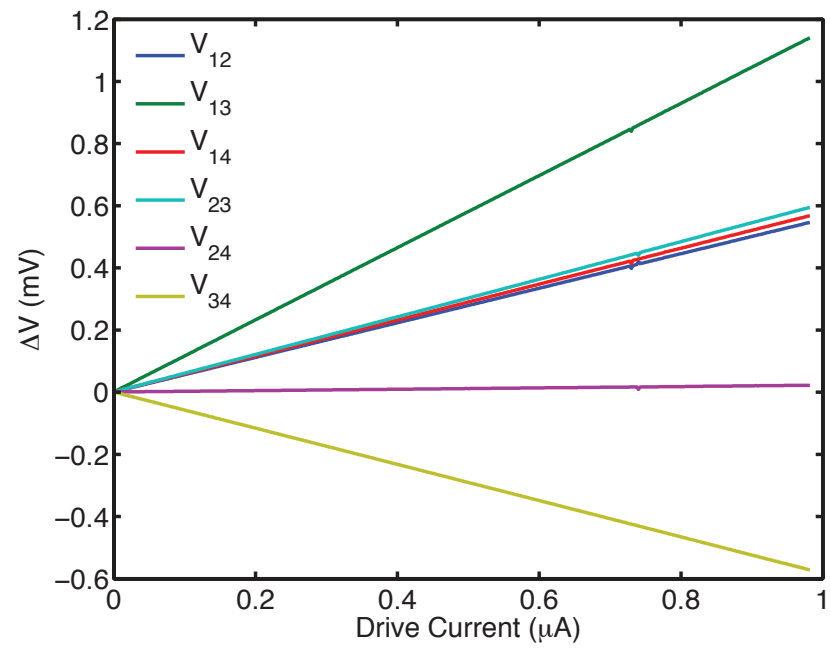

FIG. 4: The induced voltages $V_{\alpha \beta}$ between all pair of terminals $\alpha$ and $\beta$, as a function of an electrical current bias between terminal 1 and 3 .

here, the deviations from a linear-in-current voltage response are negligibly small, three orders of magnitude smaller than the linear response.

In the linear response regime, only the first harmonic of the voltage response is significant, and we can write the current as

$$
I_{\alpha}^{(1)}=\sum_{\beta \neq \alpha} G_{\alpha \beta} V_{\alpha \beta}^{(1)}, \quad V_{\alpha \beta}^{(1)}=V_{\alpha}^{(1)}-V_{\beta}^{(1)},
$$

where $I_{\alpha}^{(1)}=+(-) I$ at the injection (extraction) terminal and zero at the two floating terminals; and the superscript denotes the harmonic of the drive voltage frequency. Due to current conservation and gauge invariance, the conductances obey the sum rules $\sum_{\alpha} G_{\alpha \beta}=$ $\sum_{\beta} G_{\alpha \beta}=0$. Measurements of the $1 \omega$ voltage responses, $V_{\alpha}^{(1)}$, at $B=0$ for each of the three biasing configurations, together with the sum rules, allow us to determine all 16 elements $G_{\alpha \beta}$ of the conductance matrix $G$. Our analysis gives

$$
G(B=0)=\frac{2 e^{2}}{h}\left(\begin{array}{cccc}
-21.3 & 11.7 & 1.5 & 8.1 \\
11.5 & -25.3 & 10.5 & 3.3 \\
1.6 & 10.4 & -20.1 & 8.1 \\
8.1 & 3.2 & 8.1 & -19.5
\end{array}\right)
$$

All conductance coefficients obey $G_{\alpha \beta}>2 e^{2} / h$, demonstrating that electron transport is in the open regime.

Eq. (B2) clearly shows that $G_{\alpha \beta} \approx G_{\beta \alpha}$, as expected from the fundamental symmetry of $G$. However, microreversibility predicts a complete symmetry. To further investigate the small asymmetry apparent in Eq. (B2) we plot in Fig. 2a) in the main text the four-terminal resistances $R_{\alpha \beta, \gamma \delta}(B)=V_{\alpha \beta}^{(1)} / I_{\gamma \delta}$, with $\gamma(\delta)$ representing the current injection (extraction) terminal, as a function of magnetic field, $B$. The fluctuations observed in these 


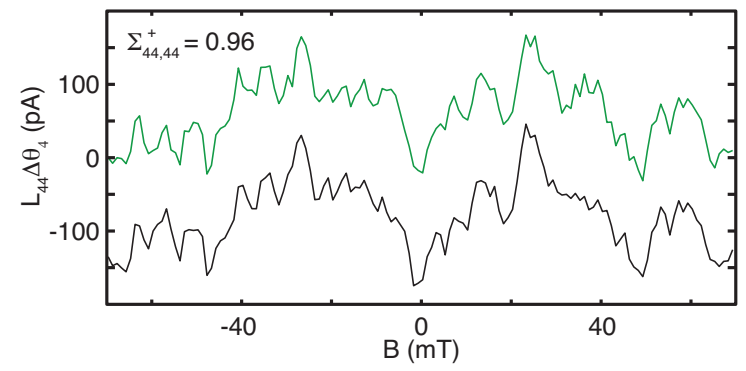

FIG. 5: Magnetic field traces of the thermoelectric coefficient $L_{44}(B)$ taken almost two weeks apart. The modified symmetry parameter $\Sigma_{44,44}^{+}$(see text), calculated for the range $-50 \mathrm{mT}<B<50 \mathrm{mT}$, gives a value close to unity, demonstrating a high degree of repeatability. The measurements were performed at a cryostat temperature of $\theta_{0}=240 \mathrm{mK}$.

traces are due to wave interference effects typical for open mesoscopic conductors. We see that the required symmetry relation $R_{\alpha \beta, \gamma \delta}(B)=R_{\gamma \delta, \alpha \beta}(-B)$ is slightly violated, in particular at the lower fields, $|B| \lesssim 50 \mathrm{mT}$, consistent with the slightly asymmetric conductance coefficients $G_{\alpha \beta}$ observed in Eq (B2). The origin of this asymmetry is not clear. We can rule out noise and nonlinear effects ${ }^{38}$ as the cause by comparing to a second measurement taken at much higher bias current, which essentially shows the same asymmetry. A magnetic sample holder can also be ruled out, as care was taken to use non-magnetic materials. Leakage currents due to the voltage probes are also found to be negligibly small. We speculate that magnetic impurities may play a role.

The typical magnitude of the magnetic field dependent oscillations of $G_{\alpha \beta}$ can be qualitatively estimated from corresponding fluctuations of the longitudinal fourterminal resistance $R_{24,24}$ in Fig. 2 in the manuscript (similar results obtained for other $R_{\alpha \beta, \alpha \beta}$, not presented). In the magnetic field range $-50 \mathrm{mT}<B<$ $50 \mathrm{mT}$ the fluctuations are of the order of a few percent, to be expected ${ }^{39}$ from a mesoscopic system in the open transport regime with a typical conductance $G_{\alpha \beta} \sim 10 e^{2} / h$, see Eq. (B2).

\section{Appendix C: Symmetry measure and reproducibility}

To quantify the degree to which the two data sets $L_{\alpha \beta}$ and $L_{\gamma \delta}$ obey the symmetry relation $L_{\alpha \beta}(B)=L_{\gamma \delta}(-B)$, we make use of Pearsons product-moment correlation coefficient, or r-correlation coefficient ${ }^{30}$, between $L_{\alpha \beta}(B)$ and $L_{\gamma \delta}(-B)$, our Eqs. (7) and (8) in the main text. The r-correlation coefficient is a well established measure to quantify the correlation between two data sets. Importantly, the r-correlation coefficient is known to be a reliable measure of correlation in the absence of outliers, i.e. extreme, isolated measurement points. We carefully investigated our data to rule out such extreme points.

To investigate the repeatability of the magnetic field traces $L_{\alpha \beta}(B)$, two traces measured almost two weeks apart are shown in Fig. 5. The degree of correlation between the two traces is quantified by the modified symmetry parameter $\Sigma_{\alpha \beta, \gamma \delta}^{+}=\sum_{B} \mathcal{L}_{\alpha \beta}(B) \mathcal{L}_{\gamma \delta}(B)$, reaching 1 for perfect correlation $\mathcal{L}_{\alpha \beta}(B)=\mathcal{L}_{\gamma \delta}(B)$.

\section{Appendix D: Linear thermal bias response}

To establish the range over $V_{H}$ where we expect a linear-in-temperature response, we used the solution of the quasi-one-dimensional heat diffusion equation ${ }^{34}$ to estimate the temperature rise in the heated channel, labelled below as the $\alpha$ 'th terminal, as a function of $V_{H}$,

$$
\theta_{\alpha}=\theta_{0} \sqrt{1+\left(\frac{V_{H}}{V_{C}}\right)^{2} \cos ^{2}(\omega t)}
$$

where $V_{C}$ is a heating channel dependent parameter. Using Eq. (D1), we can estimate the predicted Fourier components of $L_{\alpha \gamma} \Delta \theta_{\gamma}^{(2)}$ and compare them to our measured data, $\sum_{\beta} G_{\alpha \beta} V_{\alpha \beta}^{(2)}$. In this way, we have clearly established that the linear-response regime extends to about $V_{H} \approx 1 \mathrm{mV}$, which corresponds to $\Delta \theta_{\gamma}^{(2)} / \theta_{0} \approx 0.21$ to 0.75 depending on which terminal is heated.
${ }^{1}$ L. Onsager, Phys. Rev. 38, 2265 (1931).

2 M. Büttiker, Phys. Rev. Lett. 57, 1761 (1986).

3 A. D. Benoit, S. Washburn, C. P. Umbach, R. B. Laibowitz, and R. A. Webb, Phys. Rev. Lett. 57, 1765 (1986).

${ }^{4}$ H. van Houten, C. W. J. Beenakker, J. G. Williamson, M. E. I. Broekaart, P. H. M. van Loosdrecht, B. J. van Wees, J. E. Mooij, C. T. Foxon, and J. J. Harris, Phys. Rev. B 39, 8556 (1989).

5 P. N. Butcher, J. Phys.: Condens. Matter 2, 4869 (1990).

${ }^{6}$ P. Jacquod, R. S. Whitney, J. Meair, and M. Büttiker, Phys. Rev. B 86, 155118 (2012).

7 J. Matthews, D. Sánchez, M. Larsson, and H. Linke, Phys. Rev. B 85, 205309 (2012).

8 C. Zhou, S. Birner, Y. Tang, K. Heinselman, and
M. Grayson, Phys. Rev. Lett. 110, 227701 (2013).

9 G. Snyder and E. Toberer, Nature Mat. 7, 105 (2008).

10 G. Benenti, K. Saito, and G. Casati, Phys. Rev. Lett. 106, 230602 (2011).

11 K. Brandner, K. Saito, and U. Seifert, Phys. Rev. Lett. 110, 070603 (2013).

12 G. B. V. Balachandran and G. Casati, Phys. Rev. B 87, 165419 (2013).

13 D. Sánchez and L. Serra, Phys. Rev. B 84, 201307(R) (2011).

14 K. Saito, G. Benenti, G. Casati, and T. Prosen, Phys. Rev. B 84, 201306(R) (2011).

15 O. Entin-Wohlman and A. Aharony, Phys. Rev. B 85, 085401 (2012). 
16 M. Büttiker, IBM J. Res. Develop. 32, 317 (1988).

17 X. Zianni, Phys. Rev. B 75, 045344 (2007).

18 M. Dresselhaus, G. Chen, M. Tang, H. Lee, D. Wand, Z. Ren, J. Fleurial, and P. Gogna, Adv. Materials 19, 1043 (2007).

${ }^{19}$ H. Linke, W. Sheng, A. Löfgren, H. Xu, P. Omling, and P. E. Lindelof, Europhys. Lett. 44, 341 (1998).

20 D. Sánchez and M. Büttiker, Phys. Rev. Lett. 93, 106802 (2004).

21 B. Spivak and A. Zyuzin, Phys. Rev. Lett. 93, 226801 (2004).

22 C. A. Marlow, R. P. Taylor, M. Fairbanks, I. Shorubalko, and H. Linke, Phys. Rev. Lett. 96, 116801 (2006).

23 R. Leturcq, D. Sánchez, G. Götz, T. Ihn, K. Ensslin, D. C. Driscoll, and A. C. Gossard, Phys. Rev. Lett. 96, 126801 (2006).

24 D. M. Zumbühl, C. M. Marcus, M. P. Hanson, and A. C. Gossard, Phys. Rev. Lett. 96, 206802 (2006).

25 L. Angers, E. Zakka-Bajjani, R. Deblock, S. Guéron, H. Bouchiat, A. Cavanna, U. Gennser, and M. Polianski, Phys. Rev. B 75, 115309 (2007).

26 D. Hartmann, L. Worschech, and A. Forchel, Phys. Rev. B 78, 113306 (2008).

27 F. P. Esposito, B. Goodman, and M. Ma, Phys. Rev. B 36, 4507 (1987).

${ }^{28}$ L. W. Molenkamp, T. Gravier, H. van Houten, O. J. A. Buijk, M. A. A. Mabesoone, and C. T. Foxon, Phys. Rev.
Lett. 68, 3765 (1992)

29 S. A. van Langen, P. G. Silverstrov, and C. W. J. Beenakker, Superlattices and Microstructures 23, 691 (1998)

30 M. G. Kendall and A. Stuart, The Advanced Theory of Statistics, Volume 2: Inference and Relationship, Griffin (1973).

31 D. Sánchez and R. López, Phys. Rev. Lett. 110, 026804 (2013).

32 J. Meair and P. Jacquod, J. Phys.: Condens. Matter 25, 082201 (2013).

33 S.-Y. Hwang, D. Sánchez, M. Lee, and R. López, New J. Phys. 15, 105012 (2013).

34 F. Giazotto, T. T. Heikkilä, A. Luukanen, A. M. Savin, and J. P. Pekola, Rev. Mod. Phys. 78, 217 (2006).

35 H. L. Edwards, Q. Niu, and A. L. de Lozanne, Appl. Phys. Lett. 63, 1815 (1993).

36 T. E. Humphrey, R. Newbury, R. P. Taylor, and H. Linke, Phys. Rev. Lett. 89, 116801 (2002).

37 L. Hicks and M. Dresselhaus, Phys. Rev. B 47, 12727 (1993).

38 A. Löfgren, C. A. Marlow, T. E. Humphrey, I. Shorubalko, R. P. Taylor, P. Omling, R. Newbury, P. E. Lindelof, and H. Linke, Phys. Rev. B 73, 235321 (2006).

${ }^{39}$ C. W. J. Beenakker, Rev. Mod. Phys. 69, 731 (1997). 